\title{
Defying Gravity: A Novel Method of Converting Screen Orientation
}

\author{
Jaeyeon Lee and Da Young Ju \\ Yonsei University \\ \{jaeyeonlee,dyju\}@yonsei.ac.kr
}

\begin{abstract}
Current automatic screen rotation method used for most of the mobile devices is based only on the direction of the gravity rather than a user's pose. Therefore research have been performed to find a more human-centered approach based on users' viewing directions, gestures or hand grip patterns. Among these studies, the computational complexity and power consumption were main drawbacks. In this paper, a novel method considering users' hand grip pattern was proposed by utilizing sensors on the back of the mobile phone to convert the screen orientation between horizontal and vertical. The proposed method not only reflects both users' hand grip pattern and comfortableness but also has advantages in adaptability for other flat-back mobile devices, faster computational time and lower power load in comparison to conventional methods.
\end{abstract}

Keywords: mobile device interaction; screen orientation; hand grip pattern; sensor

\section{Introduction}

Current gravity-based method of automatically changing screen orientation performs well in most cases. However, recent study [7] demonstrated that there are notable discomforts when the screen orientation is different from actual viewing direction of the users since the current method based only on gravity. Therefore, research have been conducted to find a more human-centered approach in terms of users' gestures, viewing directions and hand grip patterns. The gesture-based methods $[3,13,14]$ provide the most intuitive way of converting screen orientation. They require additional cognitive actions while the gravity-based method naturally switches a screen orientation. Further, when it comes to drive the extra devices, power consumption should be one of the main drawbacks. On the other hand, the face detection technique $[1,12,7]$ makes screen orientation to adjust to the users' viewing direction that is acquired by front camera implemented in mobile devices. Since the method utilizes the embedded camera, it does not need any more circuit to operate. However, it has limitations with insufficient light condition and lack of feature points for detecting face regions. Performance of the face detection is significantly related to such external conditions. Finally, since it utilizes users' habitual behavior and robust to external environment such as light or sound, the methods based on hand grip pattern [8-11] are the most natural way of detecting appropriate screen orientation. However, several disadvantages including high power consumption and long computational time were reported in recent study. Moreover, for the portable devices, it becomes an important factors that should not be ignored the increasing discomfort caused by volume and weight. In this study, a more natural and faster implementation of the users' hand grip pattern is proposed by using three small-thin sensors on the back of the flat-back devices. 


\section{Proposed Method}

In the proposed method, the screen orientation is determined by utilizing combination of three sensors on the back of a mobile device. Experiments were conducted under the thesis that the hand grip patterns are similar within vertical or horizontal modes and far differ between the two modes. Main purpose of this experiment was to find out a feature point to distinguish users' intend to use one of the orientations.

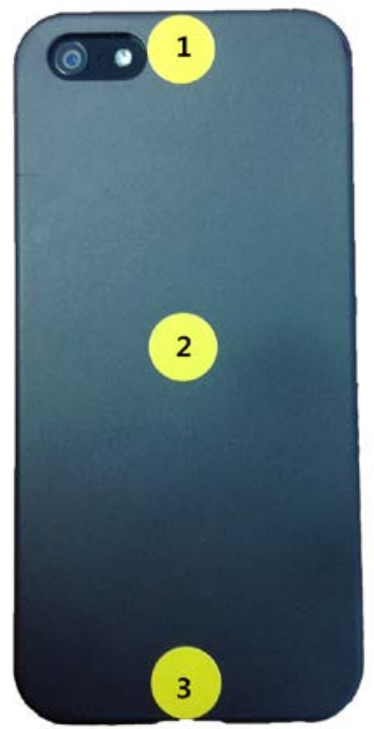

Figure 1. Mobile Phone with Three Markers on the Back $(12.5 \mathrm{~cm} \mathrm{x} 6 \mathrm{~cm})$

In Figure 1, three markers equally spaced at the center of a back of device indicate the position that sensors will be located. According to users' hand grip pattern, sensors will have characteristic binary inputs such as $\left\{\begin{array}{llll}0 & 1 & 0\end{array}\right\}$. By using inputs as a switch, the screen orientation will be decided between horizontal and vertical. If there is a key region that is dominantly affecting on mode decision, weighting or the number of sensors could be changed.

Before implementing the proposed system, experiments were conducted to convince the fore-mentioned assumption. In the experiments, subjects were suggested to look into the contents and type some information with a mobile device, iPhone 5. To observe a habitual motion, scroll, zoom and use of both hands were all allowed if it is necessary. Since tasks were repeated in two modes; horizontal and vertical, with three poses; sitting, standing and lying, total 12 pictures (Figure 2) were acquired from each subject. Two female and three male students were observed in the experiments. They were all right-handed and in age of 23, 26, 27, 25 and 25, respectively. Hand sizes were $17.5 \mathrm{~cm}, 17.5 \mathrm{~cm}, 18.5 \mathrm{~cm}, 19 \mathrm{~cm}$ and $17 \mathrm{~cm}$, respectively. All of them are completely used to the smart phones and they all responded 'yes' to the confirming question asking whether they usually bring smart phones into bed. During the subjects were following tasks, we took pictures of users' hands and analyzed the markers in images whether they were obscured or not. Since the markers represent the sensors, as shown in Figure 3, if a marker was obscured by any finger, 0 point was given to the sensors at the corresponding locations, in reverse, for a transparently viewing sticker, 1 point was given to the corresponding sensors. 


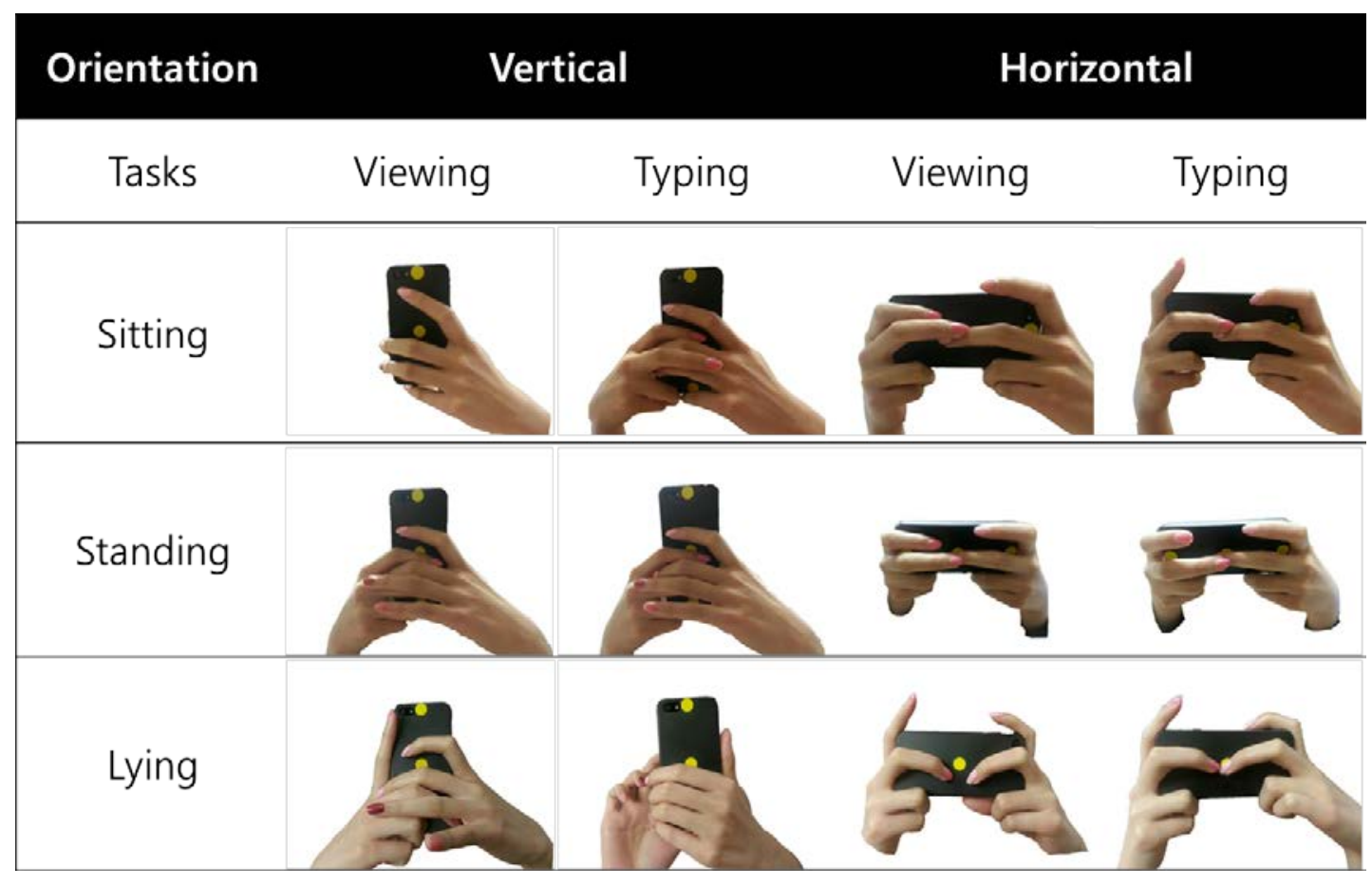

Figure 2. Data Set from one of the Subjects
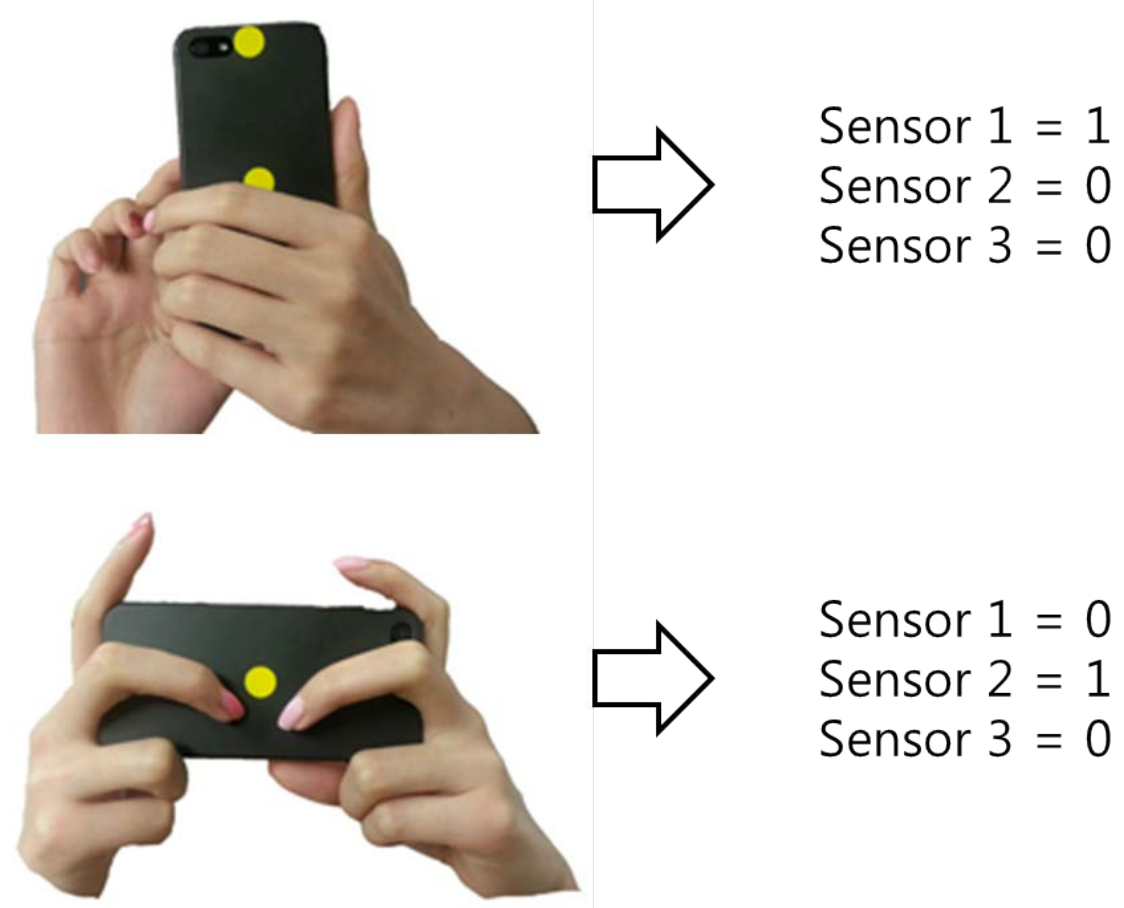

Figure 3. Examples of Hand Grip Pattern and Analysis 


\section{Experimental Results}

After scoring the data, mean and standard deviation for each sensor were calculated. In Figures 4, 5 and 6, the graph depicts mean and standard deviation for each sensor. Figures 4 and 5 represent results from horizontal cases and vertical cases, respectively. In both figures, the red lines are results of viewing task and the blue lines are those of typing task. Figure 6 is result of the total cases and the red and blue lines represent results of horizontal and vertical cases, respectively.

In horizontal mode, the scores of sensors distributed more uniformly in comparison to those of vertical case since people tended to grasp the device symmetrically. In vertical use, except only one of total 30 cases, most of the people observed to touch the bottom edge with the little finger to make it stable. Sensor 1 , which was located at the top edge of the device, scored average of 1 and standard deviation of vertical use of the phone. None of the subjects touched the top edge when he or she was using the mobile phone in vertical manner. Those two points, top and bottom edge regions, can be a key feature for detecting users' intend.

Hand grip pattern of horizontal use was little more various compared to vertical use. In both cases of horizontal and vertical uses, in a typing motion, the hand grip pattern became more similar.

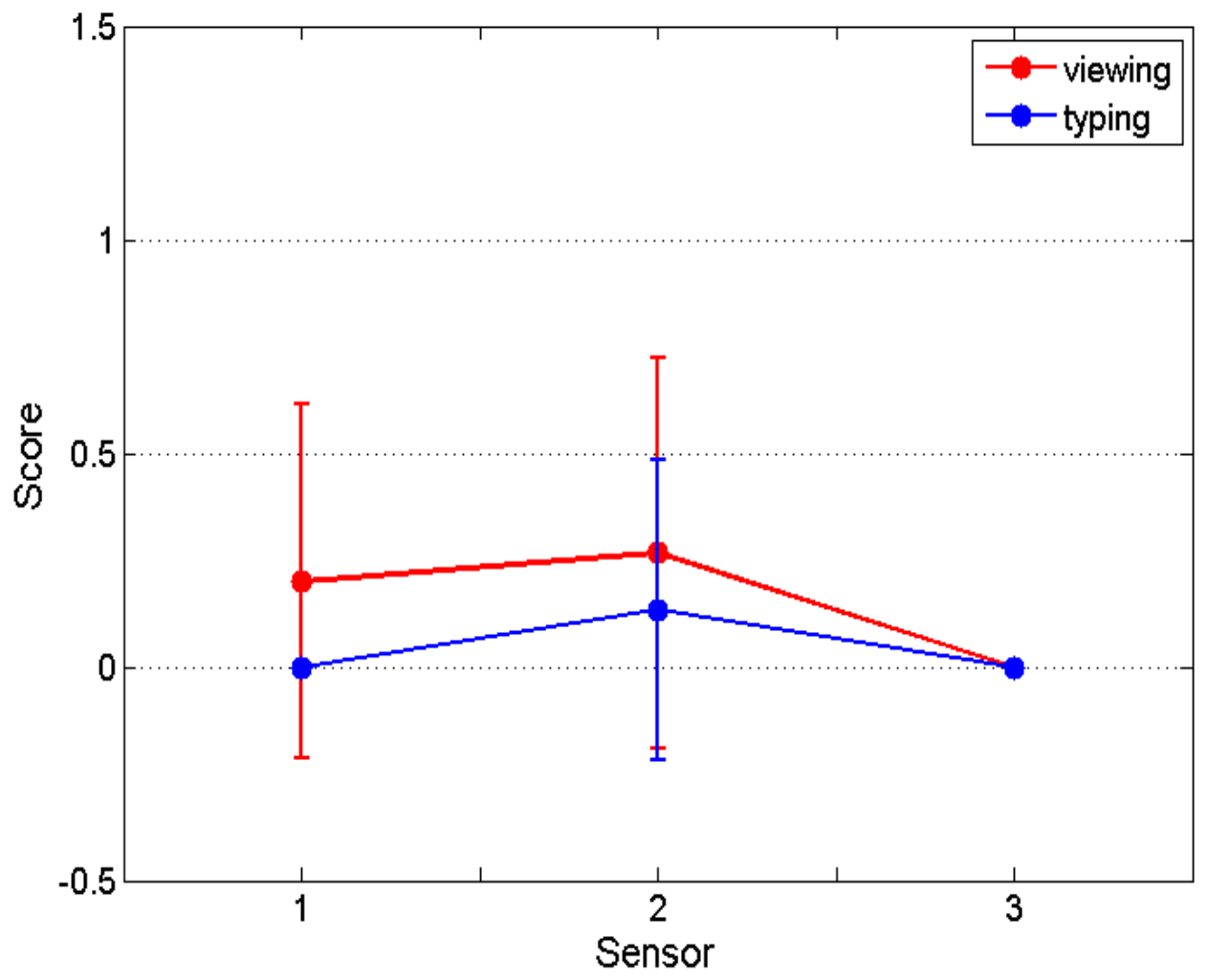

Figure 4. Scores for each Sensor Location (horizontal case) 


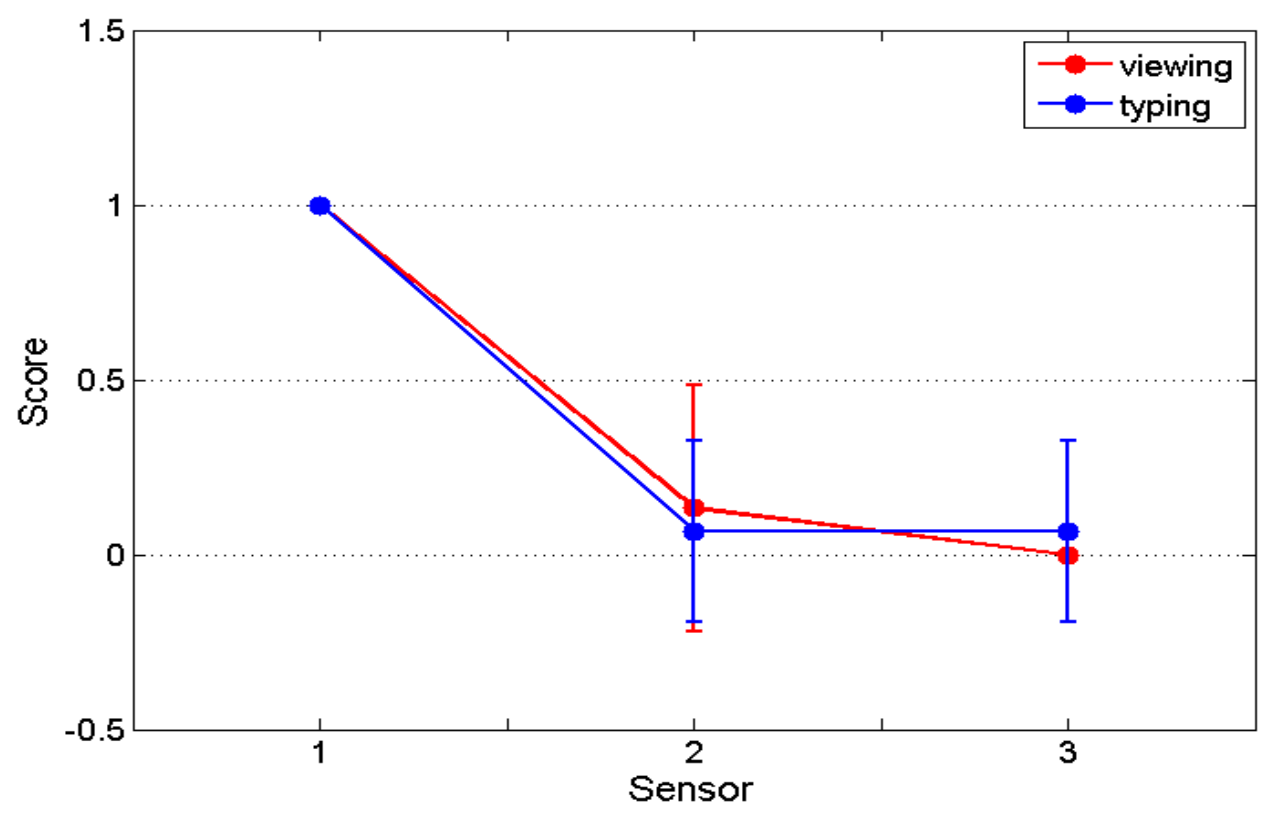

Figure 5. Scores for each Sensor Location (vertical case)

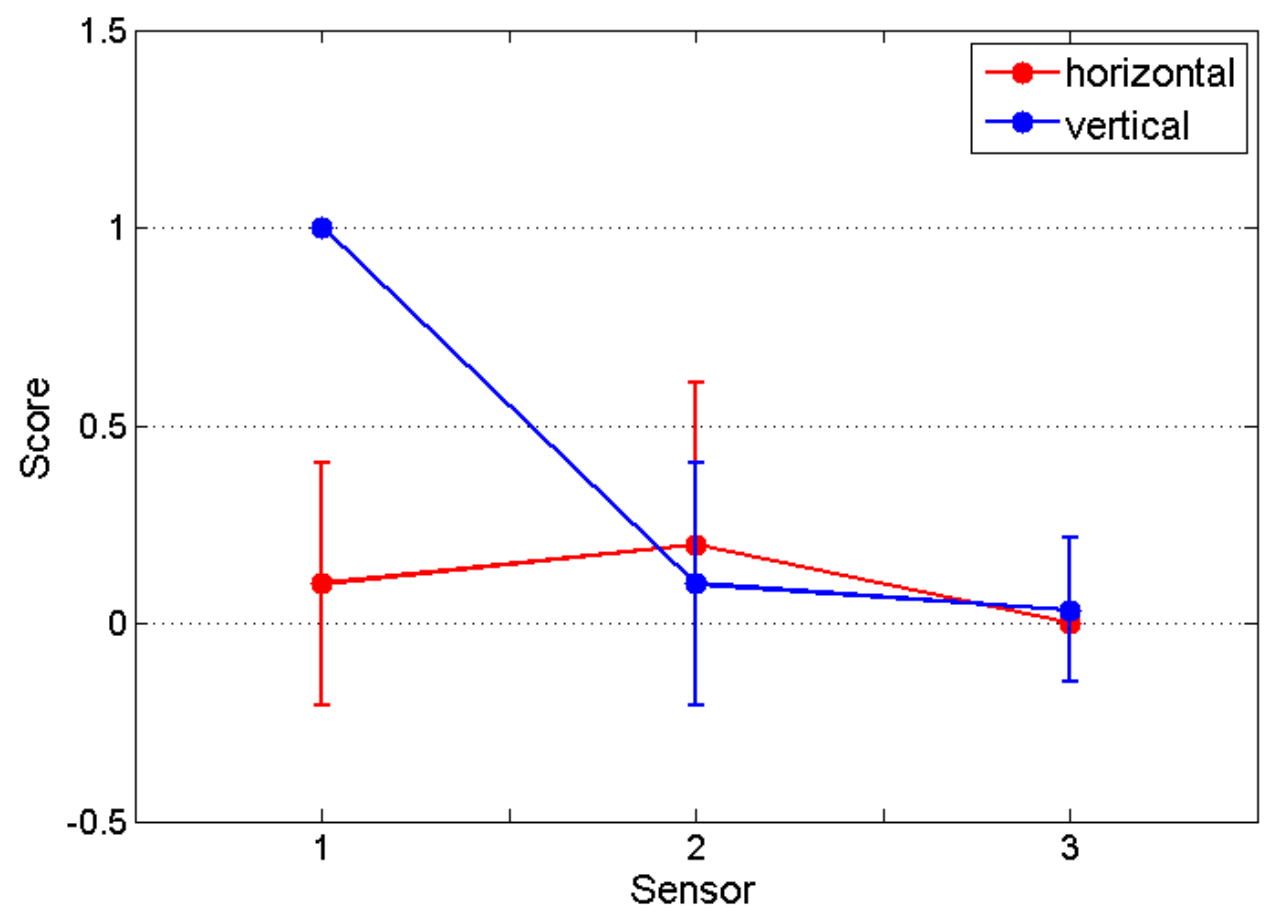

Figure 6. Scores for each Sensor Location (total case) 


\section{Conclusion and Future Works}

Since current method of changing screen orientation based only on gravity rather than users' pose, users often suffered from wrong screen orientations. Therefore studies have been conducted to find a more human-centered approach including users' gesture, face detection and hand grip pattern. In this paper, a novel method considering users' hand grip pattern was proposed. In this method, combination of three small-thin sensors located on a back of device was utilized as a switch to converting the screen orientation. Through the experimental results, hand grip patterns of vertical and horizontal screen orientation had spatial feature region such as top and bottom edge areas. With these results, proposed method provides a more natural way of converting the screen orientation since it reflects both users' habitual behavior and comfortableness. It has advantages in adaptability for other flat-back mobile devices, faster computational time and lower power consumption in comparison to conventional methods.

\section{Acknowledgements}

This research was supported by the MKE(The Ministry of Knowledge Economy), Korea, under the "IT Consilience Creative Program" support program supervised by the NIPA(National IT Industry Promotion Agency) (NIPA-2013-H0203-13-1002).

\section{References}

[1] R. Duda, P. Hart and D. Stork, “Pattern Classification”, JohnWiley \& Sons, Inc., (2001).

[2] K.-E. Kim, W. Chang, S.-J. Cho, J. Shim, H. Lee, J. Park, Y. Lee and S. Kim, "Hand grip pattern recognition for mobile user interfaces", Proceedings of the National Conference on Artificial Intelligence, (2006).

[3] A. Butler, S. Izadi and S. Hodges, "SideSight: Multi-“touch” Interaction around Small Devices”, Proceedings of the 21st annual ACM symposium on User interface software and technology, Monterey, California, USA, (2008) October 19-22.

[4] R. Wimmer and S. Boring, "HandSense- Discriminating Different Ways of Grasping and Holding a Tangible User Interface”, Proceedings of the Third International Conference on Tangible and Embedded Interaction, Cambridge, UK, (2009) February 16-18.

[5] B. Taylor and V. Michael Bove, Jr., "Graspables: Grasp-Recognition as a User Interface”, Proceedings of the $27^{\text {th }}$ international conference on Human factors in computing systems, Boston, MA, USA, (2009) April 4-9.

[6] K. Hinckley and H. Song, "Sensor Synaesthesia: Touch in Motion, and Motion in Touch", Proceedings of the 2011 annual conference on Human factors in computing systems, Vancouver, BC, Canada, (2011) May 7-12.

[7] L.-P. Cheng, F.-I. Hsiao, Y.-T. Liu and M. Y. Chen, "iRotate: Automatic Screen Rotation based on Face Orientation”, Proceedings of the 2012 ACM annual conference on Human Factors in Computing Systems, Austin, Texas, USA, (2012) May 5-10.

[8] L.-P. Cheng, F.-I. Hsiao, Y.-T. Liu and M. Y. Chen, "iRotate Grasp: Automatic Screen Rotation based on Grasp of Mobile Devices”, Proceedings of the 21st annual ACM symposium on User interface software and technology, Cambridge, Massachusetts, USA, (2012) October 7-10.

[9] M. Goel, A. Jansen, T. Mandel, S. Patel and J. Wobbrock, “ContextType: Using Hand Posture Information to Improve Mobile Touch Screen Text Entry”, Proceedings of the 2011 annual conference on Human factors in computing systems, Paris, France, (2013) April 27-May 2.

[10] L.-P. Cheng, H.-S. Liang, C.-Y. Wu and M. Y. Chen, "iGrasp: Grasp-based Adaptive Keyboard for Mobile Devices”, Proceedings of the 2011 annual conference on Human factors in computing systems, Paris, France, (2013) April 27-May 2.

[11] L.-P. Cheng, M. H. Lee, C.-Y. Wu, F.-I. Hsiao, Y.-T Liu, H.-S. Liang, Y.-C. Chiu, M.-S. Lee and M. Y. Chen, "iRotateGrasp: Automatic Screen Rotation based on Grasp of Mobile Devices", Proceedings of the 2011 annual conference on Human factors in computing systems, Paris, France, (2013) April 27-May 2.

[12] L. Jin Gu, L. Moon Heui and K. Yun Hyang, “Mobile Device Capable of Suitably Displaying Information through Recognition of User`s Face and Related Method”. US Patent US20090239579, (2009) September 24.

[13] S. Forstall and C. Blumenberg, "Portrait-Landscape Rotation Heuristics for a Portable Multifunction Device", US Patent 7,978,176 B2, (2011) July 12.

[14] B. Ording, M. Van Os and I. Chaudhri, "Screen Rotation Gestures on a Portable Multifunction Device”, US Patent 7,978,182 B2, (2011) July 12. 


\section{Authors}

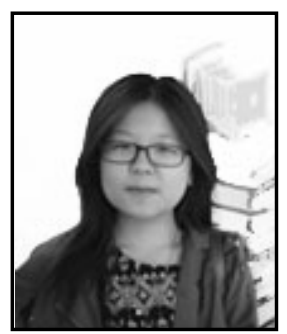

\section{Jaeyeon Lee}

2012: PhD student, Integrated Technology, Yonsei University, Korea

2010-2012: MS, Electrical Engineering, Korea Advanced Institute of Science and Technology, Korea

2006-2010: BS, Control Engineering, KwangWoon University, Korea

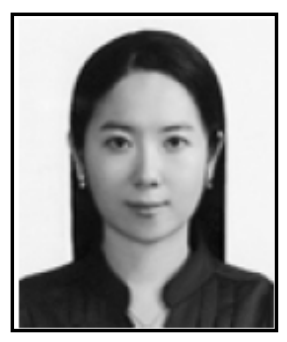

\section{Da Young Ju}

2012: Assistant Professor, Yonsei University, Korea

2002-2011: PhD, Media Technology, Sogang University, Korea

2007-2008: MA, Digital Arts, University of the Arts London, UK

2000-2002: MS, Media Technology, Sogang University, Korea

1995-2000: BFA, Painting \& Design, Hongik University, Korea 
International Journal of Smart Home Vol.7, No.5 (2013) 